\title{
Alarma digital con sensor de movimiento
}

\section{Digital alarm with motion sensor}

GONZALEZ-MONZON, Ana Lilia†*, RUEDA-MEDINA, Israel, PIÑA-ALCANTARA, Henry Christopher, y MARTINEZ-SANTIAGO, Alejandro

Tecnológico de Estudios Superiores de Jilotepec

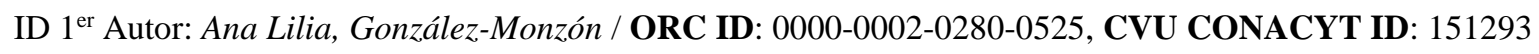

ID $1^{\text {er }}$ Coautor: Israel, Rueda-Medina / ORC ID: 0000-0003-0405-0913, CVU CONACYT ID: 153741

ID $2^{\text {do }}$ Coautor: Henry Christopher, Piña-Alcántara / ORC ID: 0000-0001-5726-2915, CVU CONACYT ID: 719620

ID $3^{\text {er }}$ Coautor: Alejandro, Martínez-Santigo / CVU CONACYT ID: IT19B186

DOI: $10.35429 /$ JOCT.2020.14.4.28.33

Recibido 16 de Septiembre, 2020; Aceptado 30 Diciembre, 2020

\section{Resumen}

La inseguridad que presentan los hogares de las comunidades de Jilotepec frente a robos a casas habitación de familias de bajo poder adquisitivo, y los altos precios de los sistemas de seguridad que ofrecen las compañías especializadas, se presenta el proyecto de realizar una alarma de digital mediante un sensor de movimiento con detección de intrusos en casa habitación. se ha desarrollado mediante la metodología CDIO (Concepción, Diseño, Implementación y operación) se utilizó la plataforma Arduino, la cual en los últimos años ha tomado mucha fuerza para la realización de proyectos de ingeniería, así como al sensor de movimiento PIR HC-SR501, que tiene un bajo costo. El sistema podrá detectar cualquier movimiento, esto con la ayuda de calor corporal de las personas, dando a conocer al usuario sobre la actividad que exista en sus hogares. Por lo tanto, ayudará a que las personas cuenten con el sistema que los haga sentir protegidas, principalmente durante la noche.

Digital, Sistema, Sensor

\begin{abstract}
The insecurity that the homes of the communities of Jilotepec present in the face of robberies to the homes of families with low purchasing power, and the high prices of the security systems offered by specialized companies, the project of making a digital alarm through a motion sensor that detects intruders in the room. It has been developed through the CDIO methodology (Conception, Design, Implementation and operation) the Arduino platform was used, which in recent years has taken a lot of strength for the realization of engineering projects, as well as the PIR motion sensor HC-SR501, which has a low cost. The system can detect any movement, this with the help of people's body heat, informing the user about the activity that exists in their homes. Therefore, it will help people to have the system that makes them feel protected, especially at night.
\end{abstract}

Digital, Sistema, Sensor

Citación: GONZALEZ-MONZON, Ana Lilia, RUEDA-MEDINA, Israel, PIÑA-ALCANTARA, Henry Christopher, y MARTINEZ-SANTIAGO, Alejandro. Alarma digital con sensor de movimiento. Revista de Tecnologías Computacionales. 2020. 4-14: $28-33$

\footnotetext{
* Correspondencia del Autor (Correo electrónico: mtra.analilia@tesji.com.edu)

$\dagger$ Investigador contribuyendo como primer autor.
} 


\section{Introducción}

Un sistema de alarma es un conjunto de dispositivos electrónicos que advierten el ingreso no autorizado en una vivienda o local, con el uso de elementos lumínicos o sonoros principalmente, existe una gran variedad de alarmas y sistemas de seguridad, tanto a nivel de funcionamiento $y$ aplicaciones como la tecnología utilizada, hoy en día estos sistemas tienen mucho más auge, pues la gente se preocupa cada vez más por su seguridad, buscando prevenir robos a casas habitación y evitar pasar por acontecimientos desafortunados.

La población ha estado en riesgo, a causa del robo a casas habitación, Las personas afectadas tienen una característica que es la falta de iluminación en sus propiedades, cabe mencionar que estos acontecimientos se dan principalmente en altas horas de la noche, cuando las personas se encuentran vulnerables, pues en la mayoría de ocasiones no logran darse cuenta de la actividad que existe dentro o fuera de casa, siendo así está una situación muy común en cualquier parte de la comunidad de Jilotepec por lo tanto La alarma con sensor de movimiento PIR, logrará detectar cualquier movimiento, para posteriormente avisar al usuario mediante un parlante sobre la actividad que exista dentro o fuera de casa.

En el primer apartado encontramos los conceptos básicos que se utilizan para el prototipo de alarma, en el segundo apartado encontramos la metodología con diseño de los planos de la base, así como el diseño en solidwork, pero también encontramos el diseño de frizzer donde está el diagrama electrónico, en el tercer apartado encontramos la programación de la placa arduino, y por último el apartado de los resultados

\section{Sistemas electrónicos}

Son circuitos electrónicos cuya misión es controlar automáticamente el funcionamiento de algunas máquinas u operadores. En todo sistema electrónico tendremos dispositivos de estos tres tipos: - Los dispositivos de entrada: generan una señal eléctrica a partir de una señal exterior de otro tipo como la temperatura, la actuación con la mano sobre un pulsador). - Los de proceso: reciben las señales de los dispositivos de entrada y deciden cual es la acción a realizar.
- Los dispositivos de salida: tienen como misión ejecutar las acciones que deciden los de proceso. Gráficamente se representan con en la siguiente Figura. (Pérez \& Sandoval, 2008).

\section{Sistemas de seguridad}

Los sistemas de seguridad son grupos de elementos instalados e intercomunicados entre sí que previenen, detectan o actúan ante intrusiones, intentos de robos y otros eventos como incendios. Por tanto, tradicionalmente, se asocian los 'sistemas de seguridad' con 'alarmas antirrobos'; sin embargo, los sistemas de alarmas son la solución moderna de mecanismos de protección que el ser humano siempre ha implementado, estos conjuntos de dispositivos varían mucho dependiendo de las características del lugar a 20 proteger, el presupuesto y si cuentan además con planes de atención y monitoreo las 24 horas.

\begin{abstract}
Alarmas
Las alarmas son un elemento de seguridad electrónica importante tanto para las empresas como para nuestras residencias. Ante la detección de una situación anormal nos pueden advertir de esta y ofrecer una respuesta inmediata con alguna acción que haya sido programada anticipadamente (ejemplo: ante la detección de una intrusión provocada por el ingreso de una persona en una zona vigilada por una alarma con sensores (detectores infrarrojos de movimientos) está hace funcionar una sirena y da aviso por radio a una central de alarmas de monitoreo o vigilancia. (Valentín, 2016).
\end{abstract}

\section{Sensores de movimiento}

Un sensor de presencia o sensor de movimiento es un dispositivo electrónico que pone en funcionamiento un sistema (encendido o apagado) cuando detecta movimiento en el área o ambiente en el que está instalado. Se utilizan con frecuencia para optimizar el consumo y la eficiencia energética de diversos sistemas como la ventilación, la iluminación o el aire acondicionado en el hogar o en la oficina, aunque también tiene aplicaciones en el ámbito de la seguridad. 


\section{Sensor infrarrojo de movimiento PIR HC- SR501}

En los sensores de movimiento, el sensor PIR consta en realidad de 2 elementos detectores separados, siendo la señal diferencial entre ambos la que permite activar la alarma de movimiento. En el caso del HC-SR501, la señal generada por el sensor ingresa al circuito integrado.

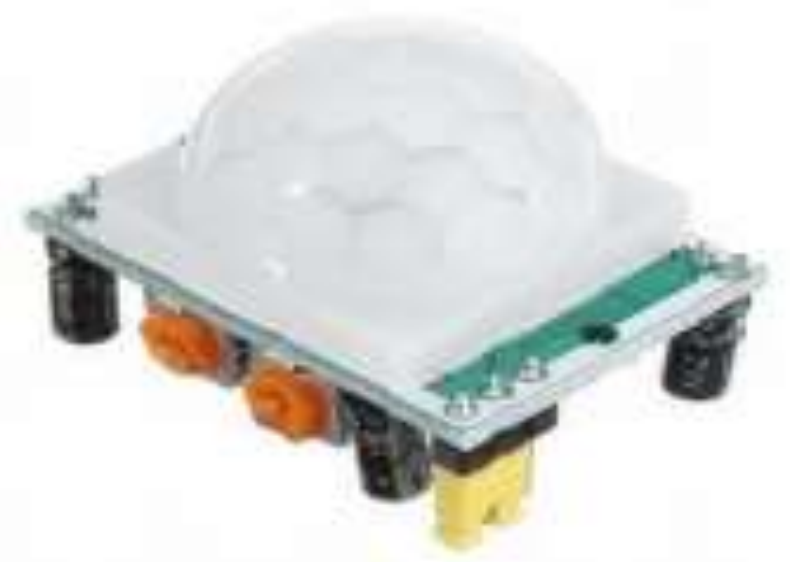

Figura 1 Sensor PIR HC-SR501

\section{Zumbador (Buzzer)}

Un zumbador es un transductor electro acústico que produce un sonido o zumbido continuo o intermitente de un mismo tono. Sirve como mecanismo de señalización o aviso y se utiliza en múltiples sistemas, como en automóviles o en electrodomésticos, incluidos los despertadores.

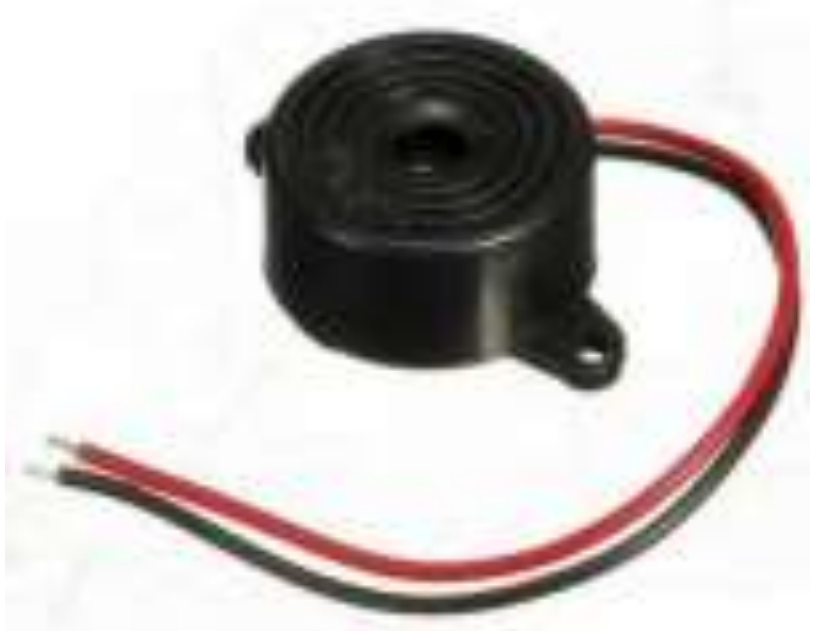

Figura 2 Zumbador

\section{Transductor}

Es el dispositivo que transforma una magnitud física (mecánica, térmica, magnética, eléctrica, óptica, etc.) en otra magnitud, normalmente eléctrica, por tanto, son especialmente importantes para que los medidores puedan detectar magnitudes físicas. Estas magnitudes, como por ejemplo temperatura, presión, humedad del aire, presión sonora, caudal, o luz, se convierten en una señal normalizada.

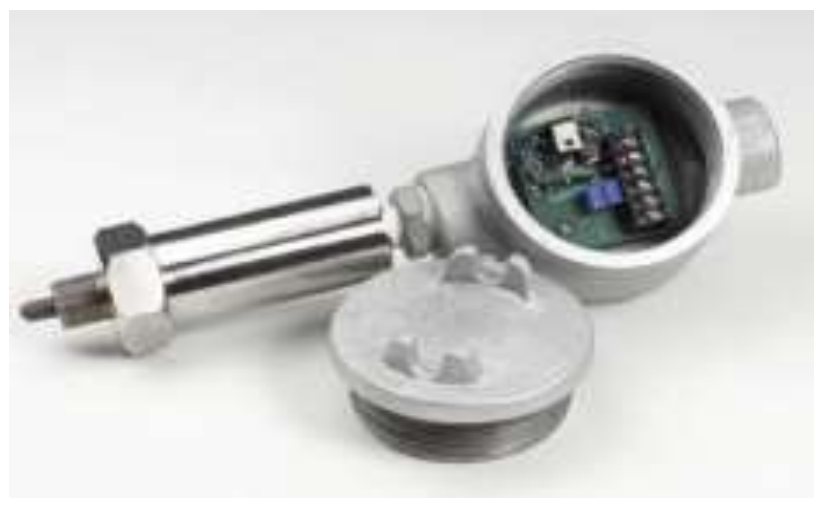

Figura 3 Transductor

\section{Arduino}

Es una plataforma de creación de electrónica de código abierto, la cual está basada en hardware y software libre, flexible y fácil de utilizar para los creadores y desarrolladores. Esta plataforma permite crear diferentes tipos de microordenadores de una sola placa a los que la comunidad de creadores puede darles diferentes tipos de uso. El control es el más importante en cualquier sistema, el uso de un Arduino facilita muchas veces hacer pruebas de componentes, y tiene un gran alcance para proyectos de todo tipo pues su interfaz es fácil de entender.

\section{Fuente de alimentación}

Componente de la instalación encargado de suministrar energía eléctrica a la central de señalización y control y a los componentes que dependen de ella. Una de estas fuentes de alimentación se encuentra en el interior de la misma central de señalización y control, aunque pueden existir fuentes de alimentación adicionales para dar servicio al resto de dispositivos que componen la instalación. 


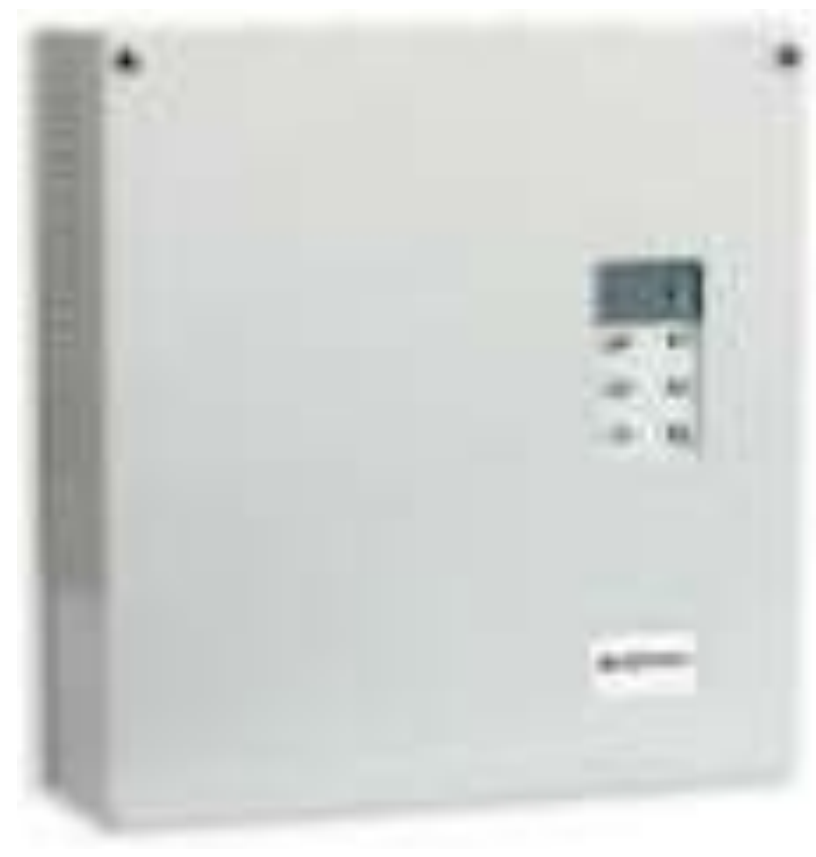

Figura 5 Fuente de alimentación

\section{Metodología a desarrollar}

La metodología (CDIO) con las siguientes actividades.

\section{- $\quad$ Investigación documental \\ - $\quad$ Diseño de planos \\ - Diseño Diagramas eléctricos \\ - Construcción de la estructura \\ - Programar el control \\ - $\quad$ Pruebas de funcionalidad}

\section{Diseño}

El diseño de la base que va postrada en la pared realizado en solidwork.

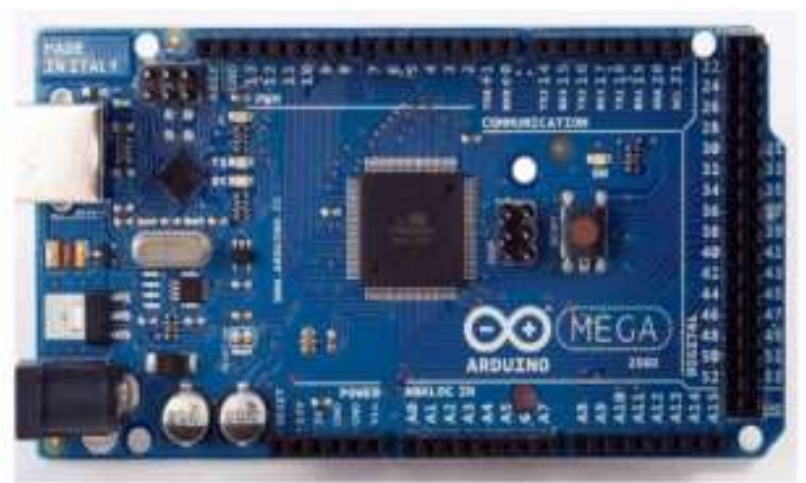

Figura 6 Arduino

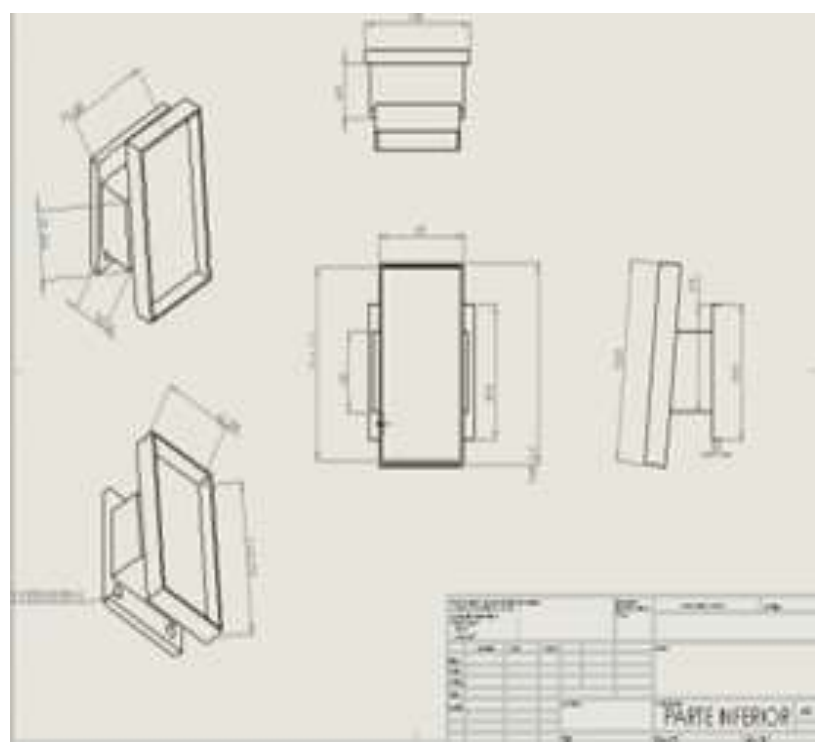

Figura 7 Plano Base pared

Diseño del Plano de la tapa de alarma para introducir

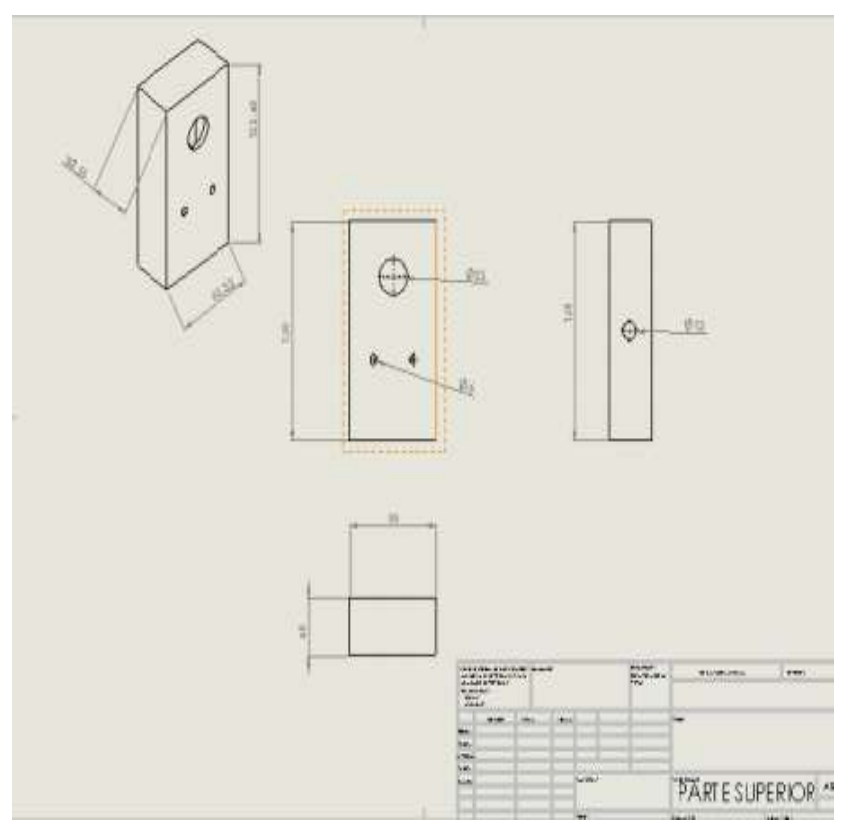

Figura 2 Plano tapa

Se realizó un diseño en SOLIDWORKS para simular el ensamblado de las piezas, además de determinar las medidas que se requieren para realizar la construcción física del prototipo. 


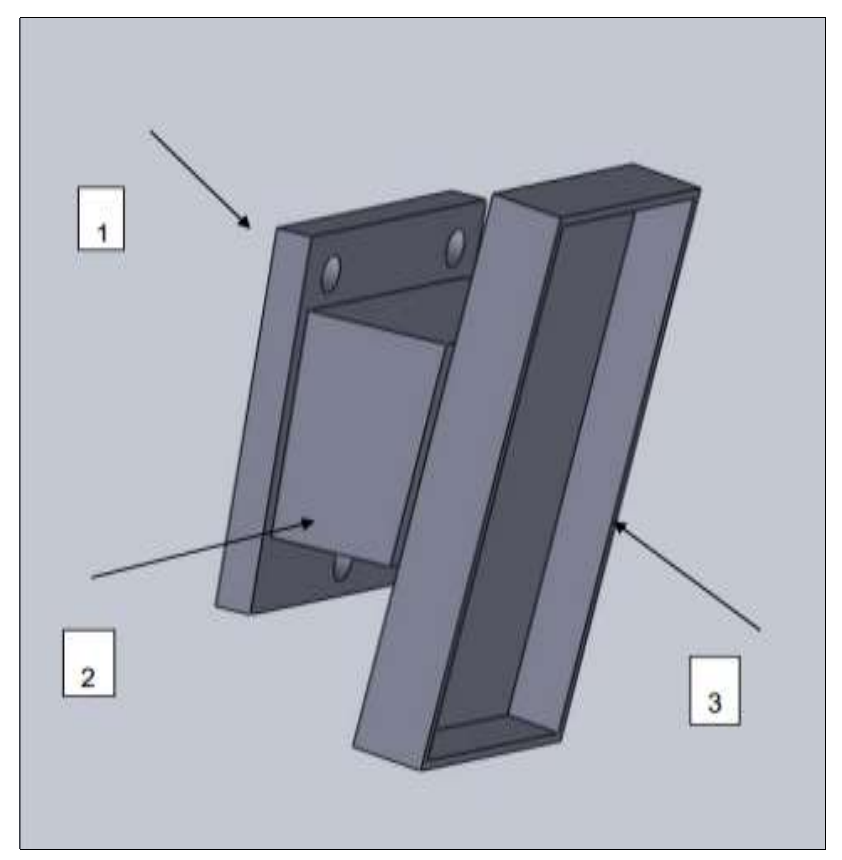

Figura 3 Base pared solidwork

Diseño de Base tapa de la alarma realizada en solidwork, Esta parte la base de la tapa, contiene 3 agujeros los cuales ayudarán para atornillar el sistema, base la cual servirá para darle el ángulo de $60^{\circ}$ al sistema, de igual forma está hecha de madera. base principal servirá para poder colocar el control del sistema.

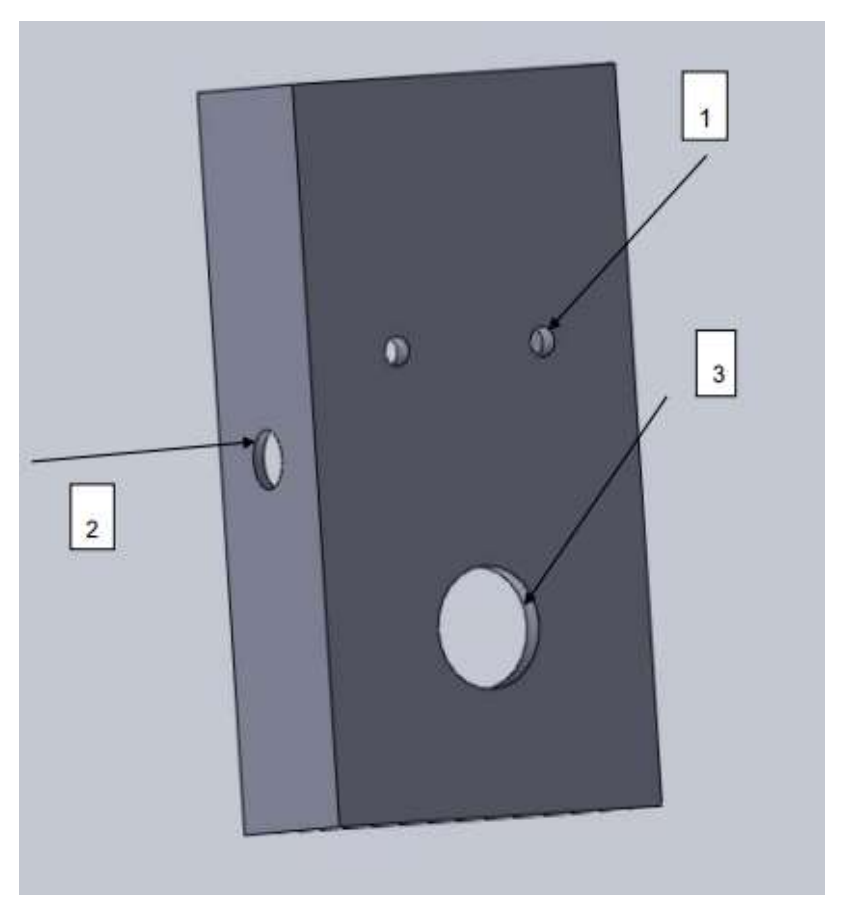

Figura 4 Tapa Base

\section{Diagrama en Fritzing}

El diagrama muestra los componentes de todo el sistema, pues es fácil entender las conexiones, además este sistema se podría hacer con Arduino Nano o un Arduino UNO.

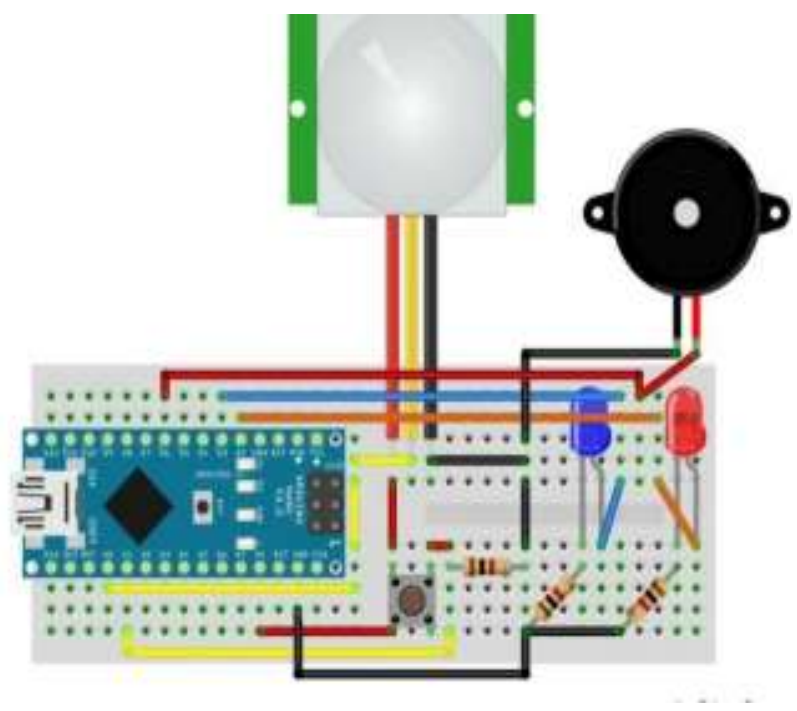

Figura 5 Diagrama Fritzing

\section{La programación}

Es de las cosas más importantes para hacer el control de algún sistema o mecanismo, pues gracias a ella se logran cumplir condiciones de las que dependen motores, sensores, pistones, $\mathrm{y}$ más.

\begin{tabular}{|c|c|}
\hline Código & Descripción \\
\hline 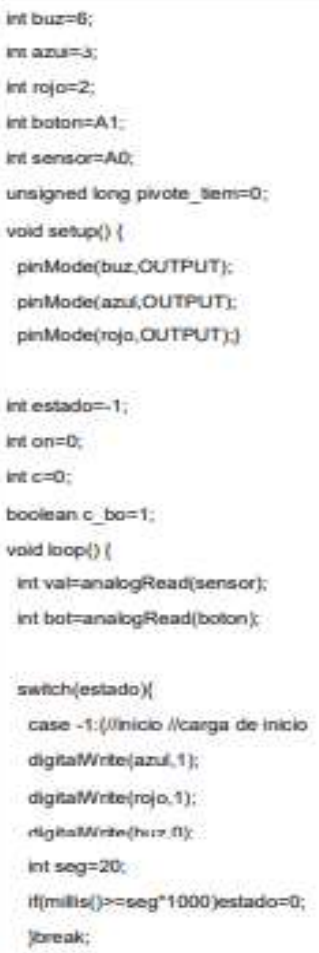 & 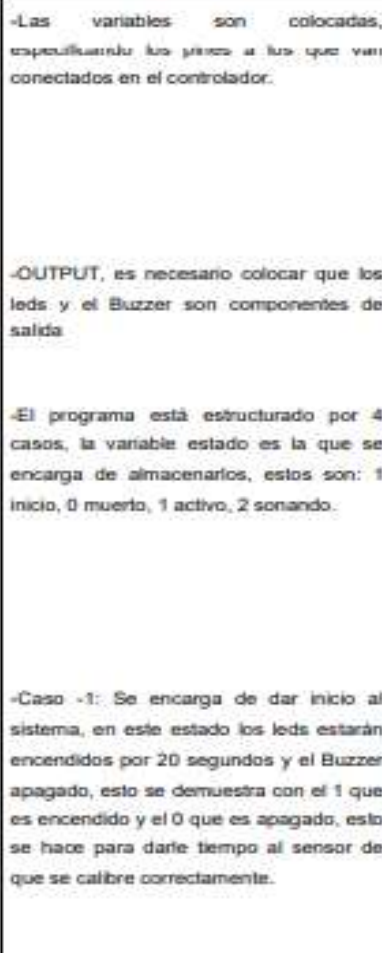 \\
\hline
\end{tabular}

\section{Resultados}

Se realizaron pruebas del sistema, específicamente se analizó el sensor infrarrojo de movimiento PIR HC-SR501 en sus dos configuraciones que tiene: la distancia a la que puede llegar a detectar y el tiempo de respuesta. 
Se le asignó el valor mínimo para el tiempo de respuesta que es de 3 segundos ya que se buscaba una rápida lectura, y para la distancia se le fue aumentando al potenciómetro para determinar qué valor era el más óptimo para lo que se buscaba, así que se configuró a 4 ms. En el instante que el sensor detecta movimiento se puede comprobar que el Buzzer tiene un sonido favorable para dar aviso al usuario de alguna posible actividad que exista, contrololado por medio de la plataforma arduino utilizando solo $5 \mathrm{v}$.

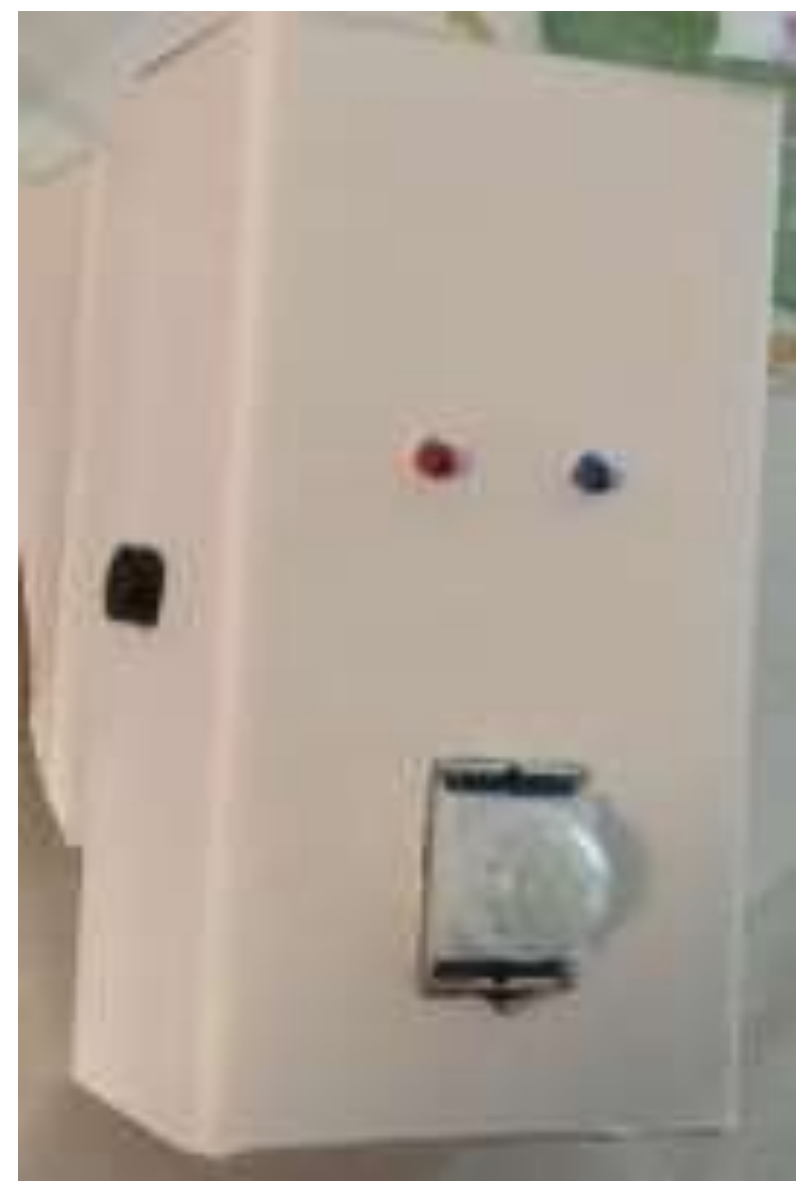

Figura 6 Alarma digital

\section{Agradecimiento}

Agradecemos al Tecnológico de Estudios Superiores de Jilotepec por el patrocinio, apoyo y facilidades otorgadas para participar en este congreso.

\section{Conclusiones}

El objetivo de este proyecto, se cumplió satisfactoriamente, ya que el sistema funcionó perfectamente, modificando el sensor a $4 \mathrm{~m}$ de detección de movimiento y su tiempo de reacción a $3 \mathrm{~s}$, al instalar el prototipo en una vivienda se observa que brinda seguridad en los usuarios.
En el instante que el sensor detecta movimiento se puede comprobar que el Buzzer tiene un sonido favorable para dar aviso al usuario de alguna posible actividad que exista, la plataforma Arduino es sin duda una herramienta muy útil para la realización del control en proyectos de cualquier tipo, además que solo se ocupan $5 \mathrm{v}$ para la alimentación del sistema, logrando poder reducir costos energéticos. El sistema puede tener un gran auge en la comunidad de Jilotepec

\section{Referencias}

Areny, R. P. (2003). Sensores y Acondicionadores de señal. Barcelona, España: marcombo.

Crespo, M., Pendino, C., \& Roldán, G. (2008). Componentes electronicos. México.

Cuzco Carrión, G. L., \& Layana Vallejo, W. A. (2012). Sistema de alarma automatizacion y control de equipos a distanca a través de linea telefónica y pagina web.

Garcia Monje, D. (2014). Diseño e implementación de un sistema de seguridad para el hogar con interfaz Android.

Pérez, M. L., \& Sandoval Valencia, J. A. (2008). TALLER 1 DE INGENIERIA ELECTRONICA Y MECATRONICA. Lima, Perú: Universidad Tecnologica Del Perú.

Raja Pérez, J. A. (2010). Diseño de sistema electrónico de alarma antiintrusos para viviendas individuales.

Reyes Ayala, J. E., \& Flores Pérez, J. L. (2016). Proyecto sensor de movimiento.

Rosales Navarro, M. Á. (2016). Diseño e implementación de alarma antirrobo. Valentín, M. C. (2016). 2.3.Sistemas de detección. En M. C. Valentín. Barcelona. 